\title{
Study of Electromagnetic Radiation and Specific Absorption Rate of Mobile Phones with Fractional Human Head Models via Green's Functions
}

\author{
${ }^{1}$ Nookala Srinivasa Rao, ${ }^{2}$ G.R. Babu and ${ }^{3}$ P.V.S. Srinivas \\ ${ }^{1}$ Department of Electronics and Communications Engineering, \\ Malla Reddy Engineering College, Secunderabad, \\ ${ }^{2}$ Department of Electronics and Communications Engineering, \\ Keshav Memorial Institute of Technology, Hyderabad, \\ ${ }^{3}$ Department of Computer Science and Engineering, \\ Geethanjali College of Engineering and Technology, Hyderabad, India
}

\begin{abstract}
Problem statement: Electromagnetic Radiation from mobile hand set is identified as one of the side effects for increasing rate of brain tumor. Due to this reason, Mobile phone industries are attentive towards safety issues of human health. Specific Absorption Rate is one of the important parameter while modeling the radiation effect on human head. Brain material with homogeneity is treated as an equivalent model of human head. The radiation caused by antennas mounted on mobile set is assumed to be monopolar. Approach: Apart from the Specific Absorption Rate, period of exposure to radiation is an extremely important parameter while assessing the effects on brain tissue. Correlation between the amount of radiation versus spherical model of brain is a complex phenomena, addressed in various simulation models. In the present work the field distribution inside the head are modeled using Dyadic Greens Functions while describing the effect of radiation pattern. Multilayered homogeneous lossy spherical model is proposed as an equivalent to head. Results: In this paper we present the depth of penetration of radiation and its effect on brain tissue. In essence the amount of electromagnetic power absorbed by biological tissues for various exposure conditions and types of emitting sources, utilizing a detailed model of the human head. Conclusion: Bio-heat equation is used to predict heat distribution inside the brain when exposed to radiation. The medium is assumed to be homogeneous, isotropic, linear, non dispersive and stationary. A critical evaluation of the method is discussed.
\end{abstract}

Key words: Electromagnetic radiation, specific absorption rate, fractional human head models, Brain material, complex phenomena

\section{INTRODUCTION}

The Radio Frequency (RF) hazard due to the electromagnetic absorption in the human head is becoming a burning problem. Extensive usage of mobile phones and other personal communication services are the main sources of Electromagnetic Radiation and absorption. In chorus with the expanding usage a question has been raised repeatedly as to whether frequent usage of such a device which radiates $\mathrm{GHz}$ electromagnetic field on the human head is unsafe. The rapid expansion has thus pushed the research toward the necessity of analyzing mobile phone for radiation performance to address the safety concern (Wong and Wiart, 2005). It is broadly accepted that mobile phones causes heating of the human organ exposed to their radiation and specifically the human head. The current exposure limits are based on Specific Absorption Rate (SAR) of the exposure heat. A SAR limit of $2 \mathrm{~W} / \mathrm{Kg}$ averaged over any contiguous $10 \mathrm{~g}$ head was recommended by the European Council (Fourie and Nitch, 2000). Most research groups studying biological effects of mobile phones have focused on two methods. Experimental measurements and Numerical techniques like FDTD. The Specific Absorption Rate (SAR) is the most appropriate metric for determining Electromagnetic effect exposure in the near field at Radio frequency source. The SAR is a metric to quantify and register localization of deposited microwave energy. The

Corresponding Author: Nookala Srinivasa Rao, Department of Electronics and Communications Engineering, Malla Reddy Engineering College, Secunderabad, India 
accuracy and reliability of a given SAR value depends on three key parameters namely tissue density, conductivity and electric field but the most significant of these is induced electric field. The induced electric field is a complex function of several physical and biological variables which include the microwave frequency, the source size and polarization, composition of the tissue including orientation. However its impossible to measure the energy absorbed in the actual human body, the measurements fluctuate from person to person due to age factor and other characteristics of the human body. In order to eliminate such problems, simulated human bodies, called biological tissue-equivalent phantoms are used (Yu and Mittra, 2004; Dey and Mittra, 1997). They simulate various electrical constants of biological tissues (e.g., the relative permittivity and conductivity) and come in various shapes from simple ones such as spheres and cubes to shapes closely resembling human bodies. Various numerical techniques are available to evaluate the Electric field intensity. Typically $40-70 \%$ of radiated RF power from a mobile phone can be absorbed by the user which depends on spectrum. Parts of human body such as neck and legs absorbs more energy in the frequency band of $100 \mathrm{kHz}-20 \mathrm{MHz}$. The influence of radiation in the band of $20 \mathrm{MHz}-300 \mathrm{MHz}$ is much higher on the surface of entire human body depending on degree of closeness. The third range of frequencies in the band $300 \mathrm{MHz}-10 \mathrm{GHz}$ reflects non-uniform absorption phenomena which is a point of our concern (Wong and Wiart, 2005). For numerical dosimetry FDTD numerical techniques is suitable because it descritises the space into a number of cells and assigns each cell a corresponding permittivity and conductivity. Also this FDTD technique offers greater flexibility in modeling the heterogeneous structures of biological organs (Yu and Mittra, 2004). Om.P.Gandhi and Cynthia M Furse were reported in theirs literature as FDTD is an efficient numerical tool for predicting the SAR and Electric field intensities in human organs ( $\mathrm{Yu}$ and Mittra, 2004). Also they reported for two age groups the near and far field strengths in the context of SAR.

The remainder of the paper is outlined as follows: Section (2) focuses on the method of calculations FDTD. Section (3) discusses the modeling of a dipole antenna in a free space. In Section (4) and Section (5), the interaction between the mobile handset and the human head has been studied as well as Electric Field and Specific absorption rate are evaluated. Section (6) Near to far field transformation is discussed. Section (7) illustrates the effect of frequency on the distribution of the SAR. Finally we present our conclusions in section (8).

FDTD formulation: In the FDTD formulation both space and time are divided in to discrete segments. space is segmented in to box shaped cells which are small in comparison with the wavelength The electric fields $(\operatorname{Ex}(\mathrm{i}, \mathrm{j}, \mathrm{k}), \operatorname{Ey}(\mathrm{i}, \mathrm{j}, \mathrm{k})$ and $\operatorname{Ez}(\mathrm{i}, \mathrm{j}, \mathrm{k}))$ are located on the edges of the box and the magnetic fields $(\mathrm{Hx}(\mathrm{i}, \mathrm{j}, \mathrm{k})$, $\mathrm{Hy}(\mathrm{i}, \mathrm{j}, \mathrm{k})$ and $\mathrm{Hz}(\mathrm{i}, \mathrm{j}, \mathrm{k}))$ are positioned on the faces as shown in Fig. 1. This orientation of the fields is known as the Yee cell (Fourie and Nitch, 2000) and is the basis for FDTD. The time is divided into small lapses where each step represents the time required for the field to travel from one cell to the next. Given an offset in space of the magnetic fields in relation to the electric fields, the values of the field in respect to time are also offset. The electric and magnetic fields are updated using a leapfrog scheme where the electric fields come first, then the magnetic ones are computed at each step in time. When many FDTD cells are combined together to form a threedimensional volume, the result is an FDTD grid or mesh. Each FDTD cell will overlap the edges and faces with their neighbors. Therefore each cell will have three electric fields that begin at a common node associated with it. The electric fields at the other nine edges of the FDTD cell will belong to other adjacent cells. Each cell will also have three magnetic fields originating on the faces of the cell adjacent to the common node of the electric field as shown in Fig. 1.

This knowledge of field values associated with the characteristics of the tissue help to determine the SAR in the tissues without requiring an invasive measure. Now we present the Maxwell's equations in three dimensions. We suppose the absence of magnetic or electric current sources and the existence of absorbing materials in the space:

$$
\nabla \times \mathrm{E}+\mu \frac{\partial \mathrm{H}}{\partial \mathrm{t}}=0
$$

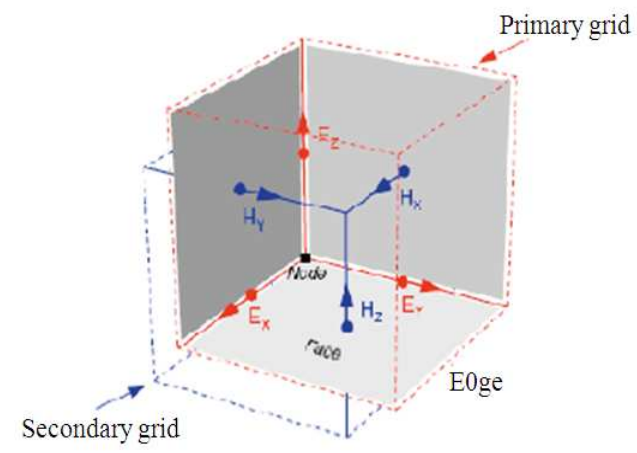

Fig. 1: Positions of the electric and magnetic Field components in a Yee cell 
$\nabla \times \mathrm{H}+\varepsilon \frac{\partial \mathrm{D}}{\partial \mathrm{t}}=\sigma \mathrm{E}$

where the displacement vector is related to the electric field through the complex permittivity:

$\varepsilon_{\mathrm{r}}^{*}(\omega)=\varepsilon r-\mathrm{j} \frac{\sigma}{\omega \varepsilon_{0}}$ by $\mathrm{D}(\omega)=\varepsilon_{\mathrm{r}}^{*} \mathrm{E}(\omega)$

$$
E_{z}^{n}\left(i, j, k+\frac{1}{2}\right)=g_{1 z} E_{z}^{n-1}\left(i, j, k+\frac{1}{2}\right)
$$$$
-g_{2 z}\left(\frac{H_{y}^{n-\frac{1}{2}}\left(i+\frac{1}{2}, j, k+\frac{1}{2}\right)-H_{y}^{n-\frac{1}{2}}\left(i-\frac{1}{2}, j, k+\frac{1}{2}\right)}{\Delta x}\right)
$$$$
-g_{2 z}\left(\frac{H_{x}^{n-\frac{1}{2}}\left(i, j+\frac{1}{2}, k+\frac{1}{2}\right)-H_{x}^{n-\frac{1}{2}}\left(i, j-\frac{1}{2}, k+\frac{1}{2}\right)}{\Delta y}\right)
$$

$\mathrm{H}_{\mathrm{z}}^{\mathrm{n}+\frac{1}{2}}\left(\mathrm{i}+\frac{1}{2}, \mathrm{j}+\frac{1}{2}, \mathrm{k}\right)=\mathrm{H}_{\mathrm{z}}^{\mathrm{n}-\frac{1}{2}}\left(\mathrm{i}+\frac{1}{2}, \mathrm{j}+\frac{1}{2}, \mathrm{k}\right)$

$-\frac{\Delta t}{\mu}\left(\frac{E_{y}^{n-\frac{1}{2}}\left(i+1, j+\frac{1}{2}, k\right)-E_{y}^{n-\frac{1}{2}}\left(i, j+\frac{1}{2}, k\right)}{\Delta x}\right)$

$+\frac{\Delta t}{\mu}\left(\frac{E_{x}^{\mathrm{n}}\left(\mathrm{i}+\frac{1}{2}, \mathrm{j}+1, \mathrm{k}\right)-\mathrm{E}_{\mathrm{x}}^{\mathrm{n}}\left(\mathrm{i}+\frac{1}{2}, \mathrm{j}, \mathrm{k}\right)}{\Delta \mathrm{y}}\right)$

$$
\mathrm{glz}\left(\mathrm{i}, \mathrm{j}, \mathrm{k}+\frac{1}{2}\right)=\frac{1-\frac{\sigma\left(\mathrm{i}, \mathrm{j}, \mathrm{k}+\frac{1}{2}\right) \Delta \mathrm{t}}{2 \delta\left(\mathrm{i}, \mathrm{j}, \mathrm{k}+\frac{1}{2}\right)}}{1+\frac{\sigma\left(\mathrm{i}, \mathrm{j}, \mathrm{k}+\frac{1}{2}\right) \Delta t}{2 \delta\left(\mathrm{i}, \mathrm{j}, \mathrm{k}+\frac{1}{2}\right)}}
$$

$$
g 2 z\left(i, j, k+\frac{1}{2}\right)=\frac{\frac{\Delta t / \sigma\left(i, j, k+\frac{1}{2}\right) \Delta t}{2 \delta\left(i, j, k+\frac{1}{2}\right)}}{1+\frac{\sigma\left(i, j, k+\frac{1}{2}\right) \Delta t}{2 \delta\left(i, j, k+\frac{1}{2}\right)}}
$$

The various components of the fields are evaluated on the basis of neighboring components of each lapse of time and each cell in the modeling area. This method works in the time domain and allows direct visualization of Electromagnetic fields.

Modeling dipole antenna in space: A simple dipole is illustrated in Fig. 2, consists of two metal arms. A dipole antenna functions with a current flow through the arms, which results in radiation. FDTD simulates a dipole in the following way. The metal of the Arms are specified by setting the Ez parameters to zero in the cells corresponding to the metal; except in place where the source is placed. This insures that the corresponding Ez field at this point remains zero as well as it would if that point were inside the metal. The antenna length was held constant at each simulation. Perfectly Matched Layer (PML) boundary conditions were employed. The source is specified by setting the Ez field in the gap to a certain value. For the FDTD simulation, dipole is fed at the center $(\mathrm{x}=\mathrm{ic} \Delta \mathrm{x}, \mathrm{y}=\mathrm{jc} \Delta \mathrm{y}, \mathrm{z}=\mathrm{kc} \Delta \mathrm{z})$ gap of length $\Delta \mathrm{z}$ with a Gaussian pulse (Taove and Hagness, 2005). So, the electric field in the gap of the dipole is:

$$
\left.\mathrm{E}_{\mathrm{z}}\right|_{\mathrm{ic}, \mathrm{n}, \mathrm{n}, \mathrm{kc}} ^{\mathrm{n}}=-\frac{\mathrm{V}(\mathrm{n} \Delta \mathrm{t})}{\Delta \mathrm{z}}
$$

Electric current: The current in the antenna at the feed point is obtained by applying Ampere's law to the surface $\mathrm{S}$ with the bounding contour $\mathrm{C}$ on the wire at (ic, jc, $k c+3 / 2)$ :

$$
\oint_{\mathrm{c}} \overrightarrow{\mathrm{H}} \cdot \mathrm{d} \overrightarrow{\mathrm{l}}=\iint_{\mathrm{s}} \overrightarrow{\mathrm{J}} \cdot \mathrm{d} \overrightarrow{\mathrm{s}}+\varepsilon_{0} \iint_{\mathrm{s}} \frac{\partial \overrightarrow{\mathrm{E}}}{\partial \mathrm{t}} \cdot \mathrm{d} \overrightarrow{\mathrm{s}}
$$

This gives the current:

$$
\begin{aligned}
& I^{\mathrm{n}+\frac{1}{2}}=\Delta x\left(\left.H_{x}\right|_{i c . j c-1 / 2, k c+1 / 2} ^{n+1 / 2}-\left.H_{x}\right|_{i c+1 / 2, j c+1 / 2, k c+1 / 2} ^{n+1 / 2}\right) \\
& +\Delta x\left(\left.H_{y}\right|_{i \mathrm{ic}+1 / 2, j c, k c+1 / 2} ^{n+1 / 2}-\left.H_{y}\right|_{i c-1 / 2, j c, k c+1 / 2} ^{n+1 / 2}\right)
\end{aligned}
$$

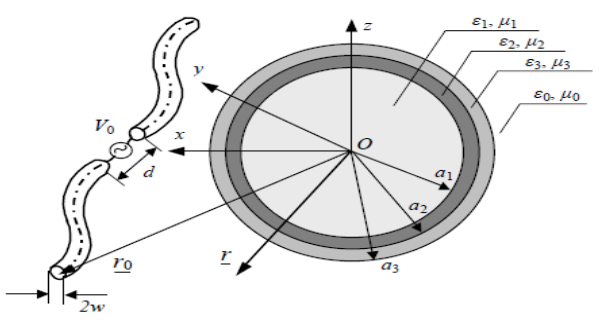

Fig. 2: Shows schematic diagram of a three layer spherical head model exposed to radiation of an arbitrarily shaped wire antenna 


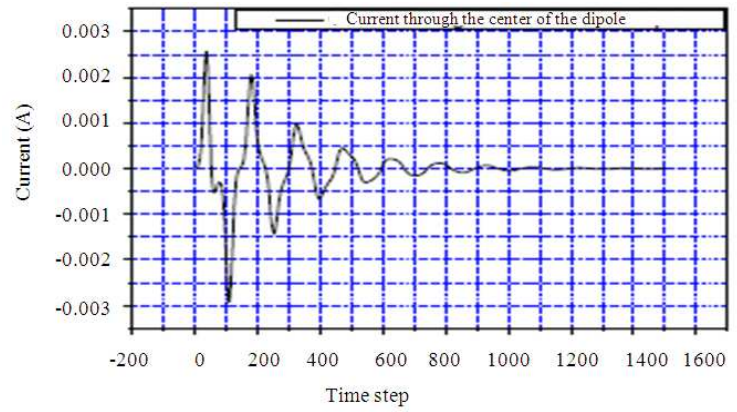

Fig. 3: Input current in terms of time I(t)

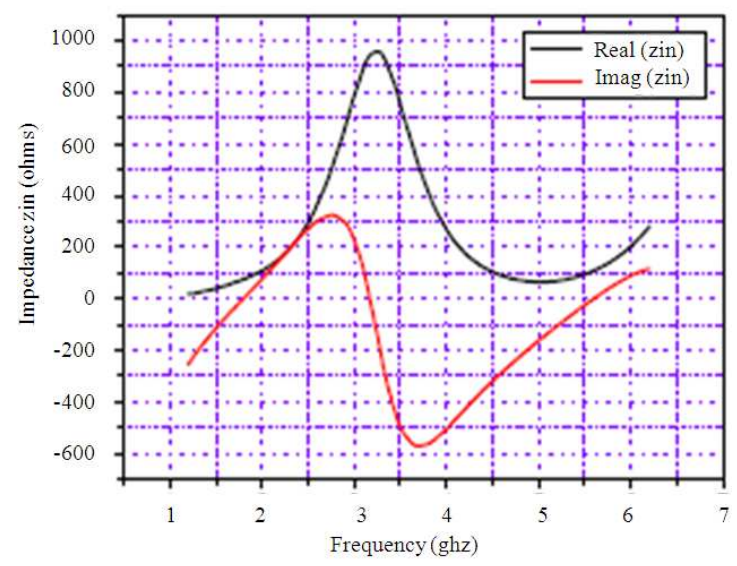

Fig. 4: Input impedance of the dipole antenna

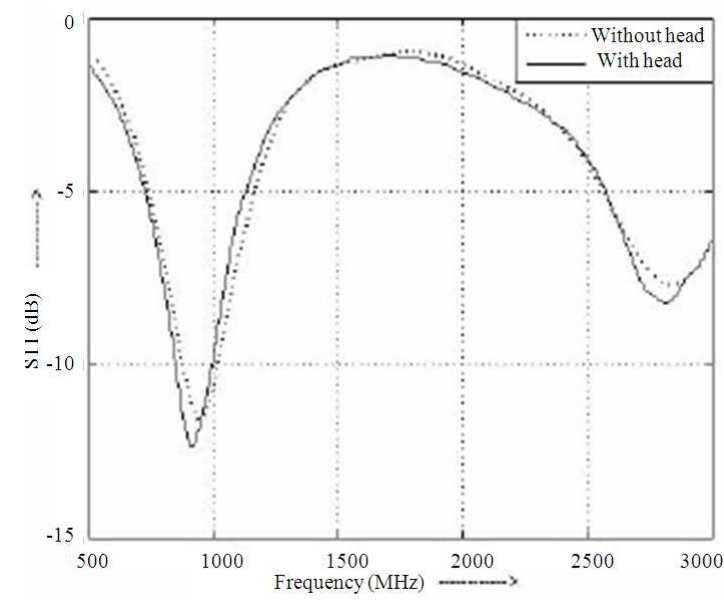

Fig 5: Shows variation of S11 versus frequency with and without head model.

The input impedance calculation: The input impedance of an antenna is a very important parameter. After the final time domain results are obtained, the current and voltage are transformed to those in the Fourier domain. The input impedance was calculated in the centre fed dipole over a range of frequencies. It is determined from the ratio of the Fourier transform of the voltage wave and that of the input current wave:

$$
Z_{\text {in }}(f)=\frac{V_{\text {in }}(f)}{I_{\text {in }}(f)}
$$

It should be noted that the time difference $\Delta t / 2$ between voltage wave and current wave is ignored since its effect is very small.

The input impedance of the dipole antenna is shown in Fig. 4.

The input impedance is well matched at $75.48+\mathrm{j} 1.12$ at the resonance frequency of $1800 \mathrm{MHz}$. In Fig. 4, the resonant frequency which is around 1.8 $\mathrm{GHz}$. was chosen as a frequency through the whole study.

The Input Return Loss (S11) and the Voltage Standing Wave Ratio (VSWR): The results of input impedance are then used to obtain the return loss characteristics of the antenna. So the reflection coefficient S11 of the half-wavelength dipole antenna is:

$$
\left|S_{11}\right|=\frac{D_{\text {FT }\left[E_{\text {ref }}\right]}}{\text { DFT }\left[E_{\text {inc }}\right]}
$$

where, $\mathrm{E}_{\text {inc }}$ and $\mathrm{E}_{\mathrm{ref}}$ are known as incident and reflected electric fields respectively. $\left|S_{11}\right|$ is computed in $\mathrm{dB}$ by:

$\left|S_{11}\right|=20 \log _{10}\left[\left|S_{11}\right|\right]$

From the calculated reflection coefficient, the Voltage Standing Wave Ratio (VSWR) can be calculated as follows:

$\operatorname{VSWR}=\frac{1+\left|\mathrm{S}_{11}\right|}{1-\left|\mathrm{S}_{11}\right|}$

The bandwidth of the antenna, which was determined by the impedance data, is the frequencies corresponding to a reflection coefficient of the antenna (less than or equal to $1 / 3$ ) that corresponds to $\mathrm{VSWR} \leq 2$. 


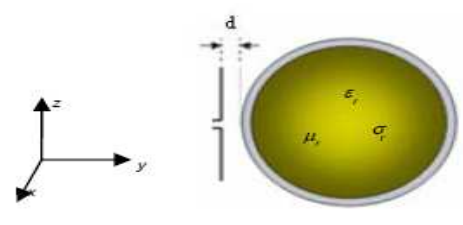

Fig. 6: Exposure of a spherical head phantom by a halfwave dipole antenna

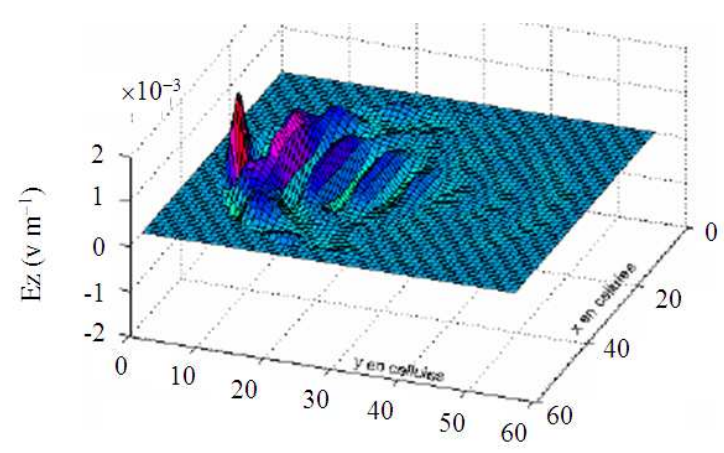

Fig. 7: The transversed electromagnetic field distribution of the dipole antenna in the homogeneous head $\mathrm{z}$ $=00 \mathrm{~mm}$

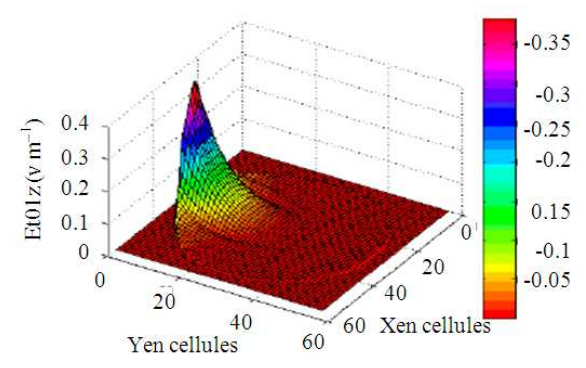

Fig. 8: The totally simulated electromagnetic field distribution of the dipole antenna in the homogeneous head $\mathrm{z}=00 \mathrm{~mm}$

Table 1: Dielectric constant, the conductivity $\sigma$ and the mass density

\begin{tabular}{llllll}
\hline & $986 \mathrm{MHz}$ & \multicolumn{2}{l}{$1800 \mathrm{MHz}$} \\
Tissues & er & $\sigma$ & er & $\sigma$ & $\begin{array}{l}\text { Density }(\rho) \\
\mathrm{kg} / \mathrm{cm}\end{array}$ \\
\hline Skin & 40.5 & 0.87 & 39 & 1.20 & 1100 \\
skull & 17.0 & 0.25 & 14.88 & 0.45 & 1845 \\
$\begin{array}{l}\text { Brain } \\
\text { Bone }\end{array}$ & 52 & 0.97 & 43.34 & 1.15 & 1045 \\
(Cortical ) 12 & 0.285 & 42.23 & 1.18 & 1200 \\
\hline
\end{tabular}

Interaction between the handset and the human head: In this section how human head is interacting with mobile handset has been studied. Simplified homogeneous head phantom model is used. Head phantom is approximated as a spherical model with radius $r=$ $10 \mathrm{~cm}$ and the relative permittivity $\varepsilon \mathrm{r}=43.5$ and conductivity $\sigma=1.15 \mathrm{~S} / \mathrm{m}$. Tissue equivalent dielectric properties were chosen according to existing literature to simulate the brain tissue at $986 \mathrm{MHz}$ and $1.8 \mathrm{GHz}$. The interaction between the mobile handset and the human head is studied from two viewpoints: first the impact of the distance between head and phone; second the effect of head type (homogeneous, heterogeneous) on the absorption and distribution of electromagnetic fields in the human head and on the radiation pattern.

The following Table 1 summarizes the dielectric constant, the conductivity $\sigma$ and the mass density of the tissues used for the calculations at 900 and 1800 MHz (Zhu et al., 2008).

NEAR-FIELDS: The near-fields have been simulated in the plane defined by $\mathrm{z}=0.0 \mathrm{~mm}$. The results are viewed in Fig. 7 and 8 for the simulations. The origin of the plane wave has been aligned in the xz-plane with the feeding point of the handset model.

Homogeneous head: The calculations were made at a frequency of $1.8 \mathrm{GHz}$. for a homogeneous spherical head of dielectric permittivity of 51.8 and a conductivity of $1.5 \mathrm{~S} / \mathrm{m}$. We calculated the distribution of the electric field in the near head near the antenna. This latter is located at a distance of $5 \mathrm{~mm}$ at the side of the spherical head. To see the effect of the position of the antenna on the radiation pattern we have traced the appearance of the reflection coefficient S11 for several distances between the header and the phone.

From Fig. 9 we can say that the radiation of the antenna depends on the distance between the phone and the header. Hence, we can conclude that there is a coupling between head and the antenna. In Table 2, the results of the calculated driving point input impedance Zin of the dipole antenna are presented for each position of the handset in front of the homogeneous human head phantom.

Table 2 Results of the driving point input impedance, VSWR and the input return loss (S11) at each distance $\mathrm{d}(\mathrm{cm})$ between axis of dipole antenna and the outer surface of the homogeneous human head phantom.

The VSWR for each case is then determined in respect to the free space input impedance. In this case the antenna input impedance changes drastically and the input power to antenna decreases considerably. In the presence of the human head, the resonance frequency is detuned approximately $5 \%$ at GSM frequencies. 


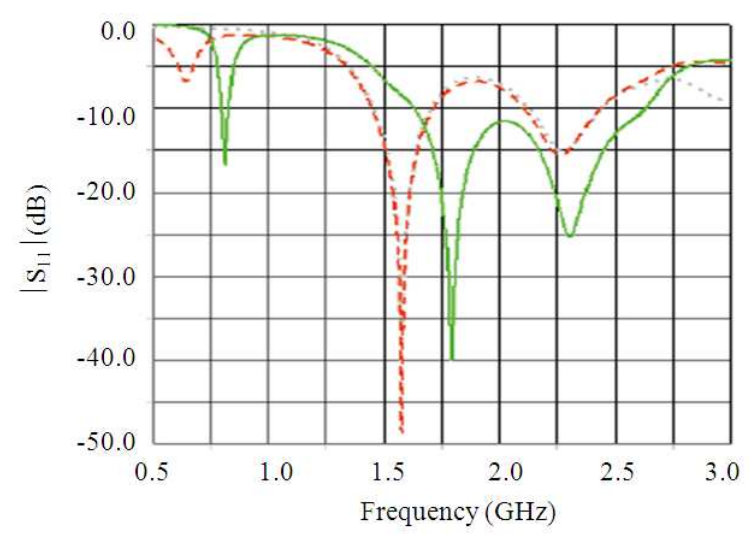

Fig. 9:Input return loss (S11) of the antenna for different values of $d$

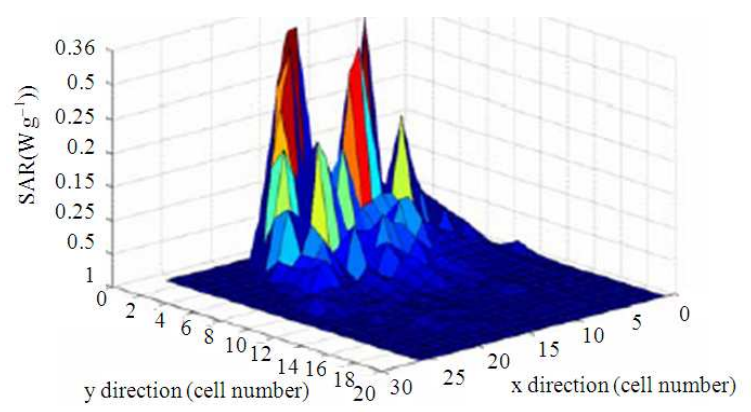

Fi. 8: The simulated electromagnetic field distribution of the dipole antenna in the heterogenous Head for $\mathrm{z}=00 \mathrm{~mm}$

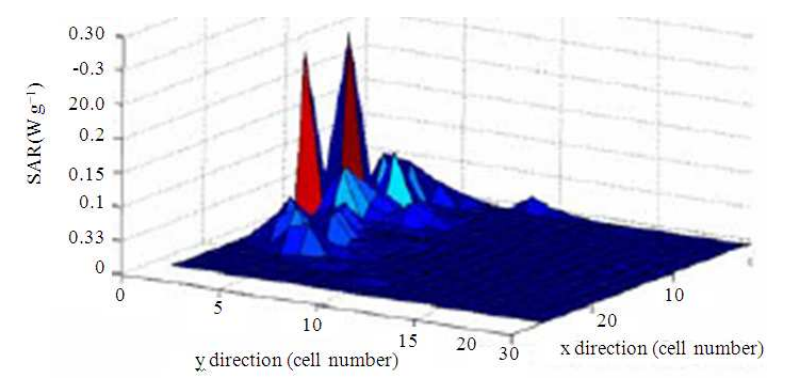

Table 2: Calculated driving point input impedance Zin

\begin{tabular}{lllll}
\hline $\mathrm{d}(\mathrm{cm})$ & $\operatorname{Re}(\mathrm{z})$ & $\operatorname{Im}(\mathrm{Zi})$ & VSWR & Abs(S11)dB \\
\hline 1.00 & 40.2 & 6.19 & 1.7888 & -9.998 \\
1.60 & 47.08 & 17.98 & 1.6777 & -10.98 \\
2.00 & 55.01 & 20.91 & 1.5550 & -11.76 \\
2.80 & 71.00 & 22.76 & 1.4000 & -14.98 \\
3.00 & 76.55 & 15.67 & 1.2000 & -18.09 \\
Free space & 75.04 & 1.13 & 1.0450 & -30.87 \\
\hline
\end{tabular}

The presence of the human head also increases the input impedance of the dipole. Hence the impedance behavior of the GSM dipole shows quite a strong dependence on the surroundings.
Heterogeneous head: A spherical heterogeneous Human head model was utilized. This phantom consisted of three layers with materials simulating the human head structure whose outer diameter is identical to that of homogeneous sphere previously used. In Table 1, the type of each layer and its corresponding relative permittivity, conductivity and density are depicted according to. In Fig. 7, 8 and 10 we see that the highest values of the electric field occur near the antenna. The magnetic field reaches its maximum value above the start of the antenna wire, near the feeding point, where the current reaches its maximum value.

SAR evaluation: The radiation source of the cellular phone was modeled by an equivalent dipole antenna. After having obtained the induced electric field by the FDTD method, the local SAR in W/Kg for:

$\operatorname{SAR}(i, j, k)=\frac{\sigma(i, j, k)|E(i, j, k)|^{2}}{2 \rho(i, j, k)}$

$\mathrm{E}$ is the electric field magnitude in $\mathrm{V} / \mathrm{m}, \sigma$ is the material conductivity in $S / m$ and $\rho$ is the mass density in $\mathrm{kg} /$ cubic metres.

Homogeneous head: An half wave dipole of $77 \mathrm{~mm}$ irradiating at $1800 \mathrm{MHz}$ is placed $10 \mathrm{~mm}$ away from the outer surface of the spherical human head. The values of SAR (or electric fields) are the highest in tissues around the antenna. These SAR values decrease rapidly when one gets away from radiotelephone antenna. As a result the tissues around the ear are most exposed to electromagnetic fields.

Heterogeneous head: Energy distribution of local SAR values are obtained directly from electric field distribution via computer simulations. Figure 12 and 13 shows the SAR absoption is dominating in the skin of the head and the surface of skull. Due to poor conductivity of the skull, energy absorption is low and variations in dielectric properties of related tissues (Rhattoy et al., 2005).

We can state that the maximum absorption occurs at the point where the phone is closer to the head. Using the relations $\mathrm{D}=\varepsilon . \mathrm{E}$ and $\frac{\sigma \mathrm{E}^{2}}{\rho}=\mathrm{SAR}$, the ratio between the SAR values in these tissues under a uniform field distribution is around 0.35. In addition, the maximum SAR values are substantially higher for the heterogeneous model of head. 


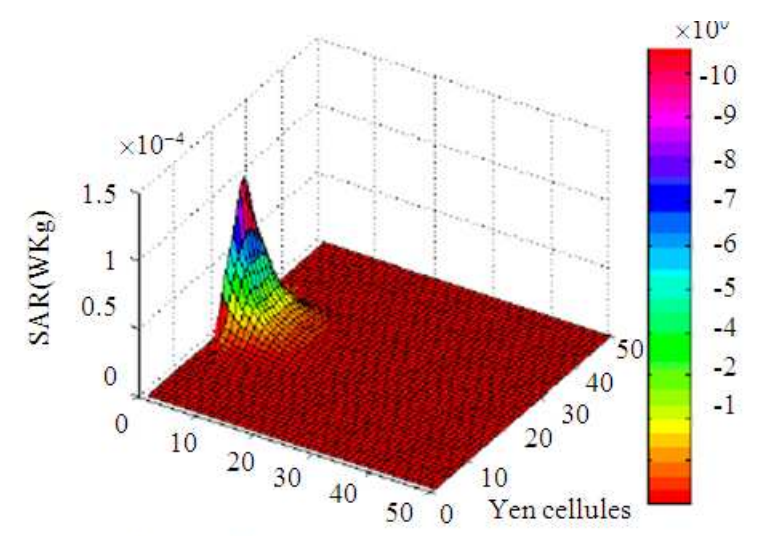

Xen cellules

Fig. 11: The simulated SAR distribution of the dipole antenna in the homogeneous Head for $\mathrm{z}=00 \mathrm{~mm}$

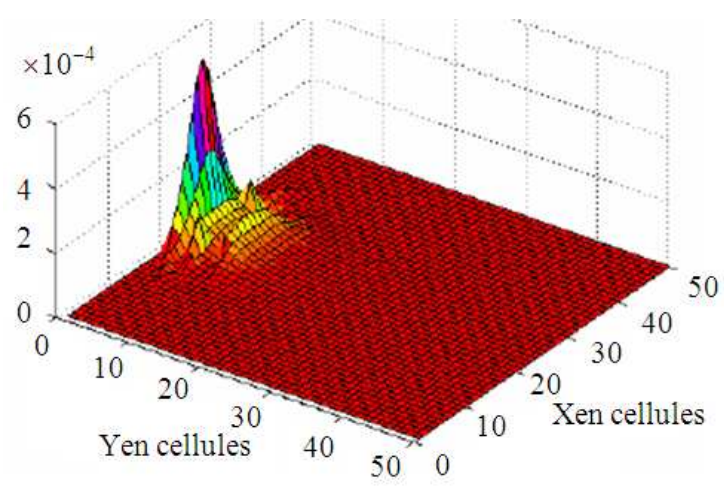

Fig. 12: The simulated SAR distribution of the dipole antenna in the heterogeneous Head for $\mathrm{z}=00 \mathrm{~mm}$

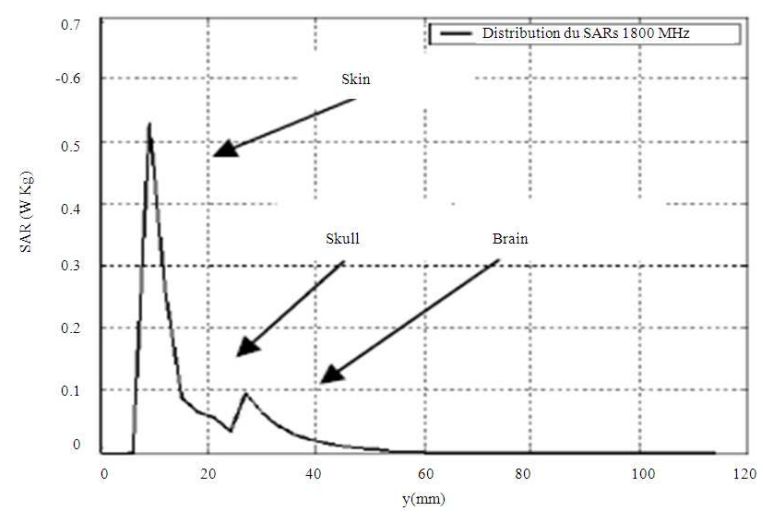

Fig. 13: SAR variation as function of the transverse distance

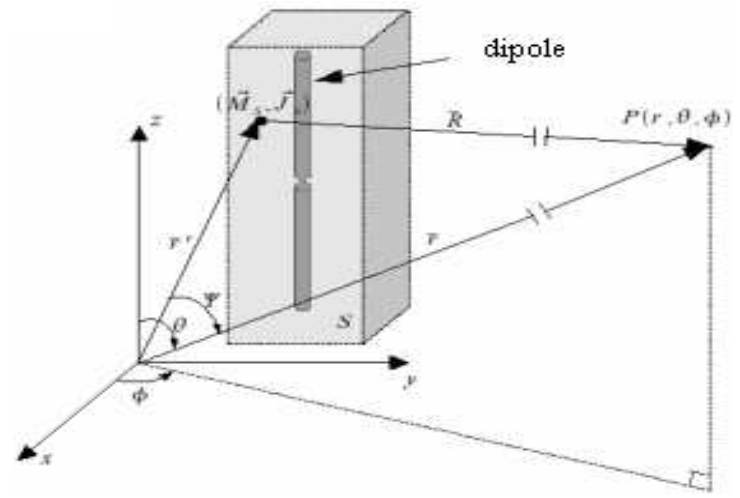

Fig. 14: The virtual surface used for the nearto-far field transformation and the coordinate system used for its calculation

Near to far field transformation: While using FDTD method, the provided data are near fields. Therefore, these near fields are transformed to far fields. Then, the far fields are used to calculate the radiation pattern. First, we work within the frequency domain and assume that the analyzed antenna is surrounded by the enclosed surface S. Then, let's assume that this closed surface has the local unit outward normal vector $n$.

Thus, the electric and magnetic current densities can be written as follows:

$$
\begin{aligned}
& \overrightarrow{\mathrm{J}_{\mathrm{s}}}=\overrightarrow{\mathrm{n}} \times \mathrm{H} \\
& \overline{\mathrm{M}_{\mathrm{s}}}=\overrightarrow{\mathrm{n}} \times \mathrm{E}
\end{aligned}
$$

Where:

$r=$ The position of the observation point $(x, y, z)$

' $r=$ The Position of the source point on $S\left(x^{\prime}, y^{\prime}, z^{\prime}\right)$

$\Psi=$ The angle between $r$ and $\mathrm{r}$

$\mathrm{E}$ and $\mathrm{H}$ are electric and magnetic fields that propagate on the surface. Later, we can define the time harmonic vector potentials $\mathrm{N}$ and L (Rhattoy et al., 2005):

$\overrightarrow{\mathrm{L}}=\int_{\mathrm{s}} \overline{\mathrm{M}} \mathrm{e}_{\mathrm{x}}^{\mathrm{jkrr}{ }^{\prime}}$ and $\overrightarrow{\mathrm{L}}=\int_{\mathrm{s}} \overline{\mathrm{M}} \mathrm{e}_{\mathrm{x}}^{\mathrm{jkrr}} \mathrm{ds}$

$\mathrm{j}=-1$, $\mathrm{k}$ is the numerical wave number, $r$, is the unit vector to the far field point and r' is the vector to the source point of integration. To obtain the far-field information from the equivalent currents, it is necessary to integrate them over each of the six faces of the virtual box, here labeled surface $S$. That integration can be done by using the following pair of vector: 
$\mathrm{N}_{\theta}=\iint_{\mathrm{S}}\left(\left(\mathrm{J}_{\mathrm{x}} \cos \phi+\mathrm{J}_{\mathrm{y}} \sin \phi\right) \cos \theta-\mathrm{J}_{\mathrm{z}} \sin \theta\right) \mathrm{e}^{\mathrm{jkr} \mathrm{r}^{\prime} \cos \psi} \mathrm{ds}$

$\mathrm{N}_{\phi}=\iint_{\mathrm{S}}\left(\mathrm{J}_{\mathrm{x}} \sin \phi+\mathrm{J}_{\mathrm{y}} \cos \phi\right) \mathrm{e}^{\mathrm{jkr} r^{\prime} \cos \Psi} \mathrm{ds}$

$\left.\mathrm{L}_{\theta}=\iint_{\mathrm{S}}^{\left(\left(\mathrm{M}_{\mathrm{x}} \cos \phi+\mathrm{M}_{\mathrm{y}} \sin \phi\right)\right.} \cos \theta-\mathrm{M}_{\mathrm{z}} \sin \theta\right) \mathrm{e}^{\mathrm{jkr} \cos \Psi} \mathrm{ds}$

$\mathrm{L}_{\phi}=\iint_{\mathrm{S}}\left(-\mathrm{M}_{\mathrm{x}} \sin \phi+\mathrm{M}_{\mathrm{y}} \cos \phi\right) \mathrm{e}^{\mathrm{jkr} \mathrm{cos}^{\prime} \Psi} \mathrm{ds}$

Finally the far electric field can be calculated by the following relation:

$$
\begin{aligned}
& E \theta=\frac{j k e^{-j k r}}{4 \pi r}\left(L_{\phi}+\eta_{0} N_{\theta}\right) \\
& E \phi=\frac{j k e^{-j k r}}{4 \pi r}\left(L_{\theta}+\eta_{0} N_{\phi}\right)
\end{aligned}
$$

the radiation pattern is given by:

$$
\mathrm{G}(\theta, \phi)=-\frac{\mathrm{k}^{2}}{8 \pi \eta_{0} \mathrm{P}_{\mathrm{e}}}\left(\left|\mathrm{L}_{\phi}+\eta_{0} \mathrm{~N}_{\theta}\right|^{2}+\left|\mathrm{L}_{\theta}+\eta_{0} \mathrm{~N}_{\phi}\right|^{2}\right)
$$

Where:

$\mathrm{P}_{\mathrm{e}} \quad=$ The input power antenna $\sqrt{\mu \mathrm{o} / \varepsilon \mathrm{o}}=\eta_{0}=$ The free space

The radiating patterns were simulated in the $\mathrm{XY}$ plane (plan $\mathrm{E}$ ) and in the $\mathrm{YZ}$ plane (H plane) for the dipole alone at the center frequency of the band $(1.8 \mathrm{GHz})$.

Figure 16: Radiation patterns for linear dipole antenna radiating in the presence of homogeneous spherical head model. Figures 15 and 16 show the effect of the head model in the XY plane (plane E) and in the YZ plane (H plane). We can see that the head blocks the radiation patterns in the head direction. In the YZ plane the radiation in halfspace where the head is situated is affected seriously. So in the XY plane the radiation more decreased towards the head's direction.

Effect of frequency: To see the effect of the frequency on the distribution of the SAR, we have drawn the SAR's profile for the two used frequencies $(900 \mathrm{MHz}$ and 1800 $\mathrm{MHz}$ ) and a homogeneous head.

Figure 17 illustrates the profile of the maximum local SAR across the homogeneous spherical head model. The distance was measured from the point of the source closest to the head model. The SAR values were normalized to the maximum to show the effect of the frequency. We can observe that the SAR decreased faster in thehigher frequency range as expected due to the weak depth penetration.

Thermal simulations: After SAR has been calculated and recorded it is utilized as source term in the transient bio heat equation (Zhu et al., 2008):

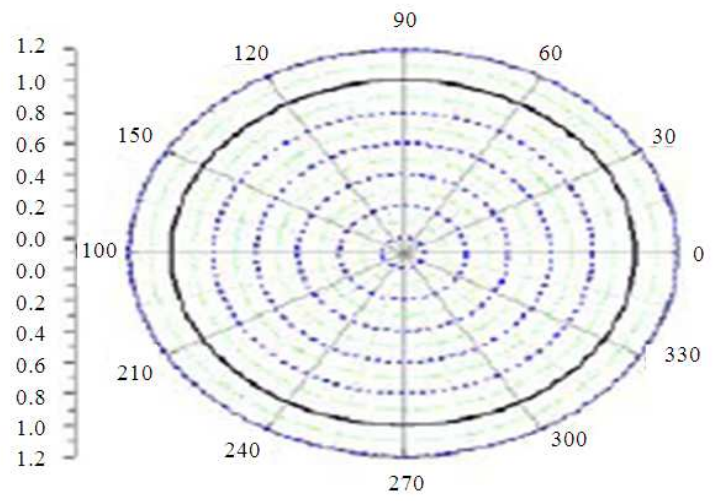

Fig. 15: a, $\theta=90 \mathrm{deg}$, xy plane.

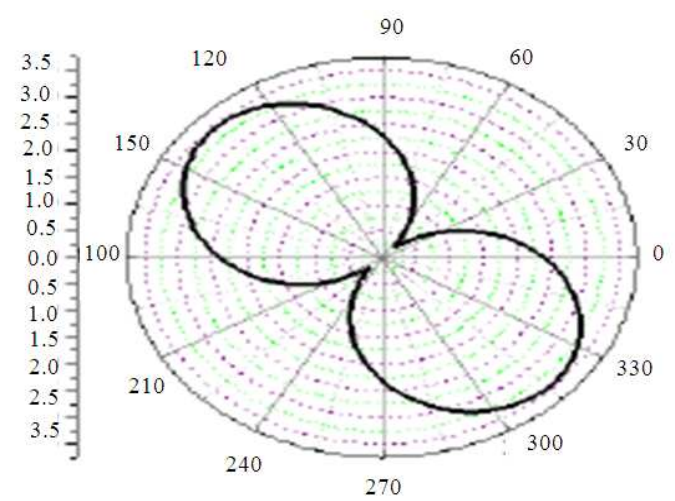

Fig. 15: b, $\varphi=90 \mathrm{deg}$, yz plane.

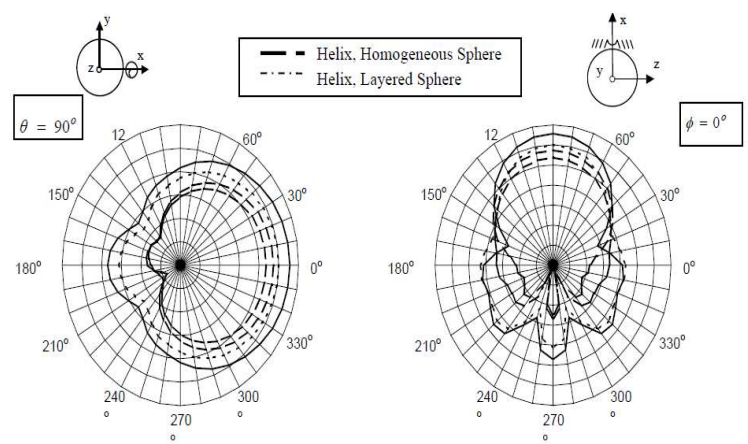

Fig. 16: b, $\varphi=0$ deg, and $\theta=90 d e g$. yz plane. 


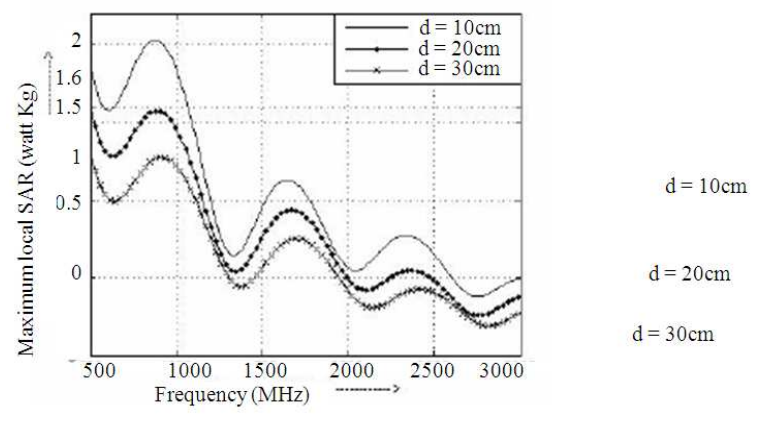

Fig. 17: SAR normalized profile through a homogeneous spherical head for two different frequencies

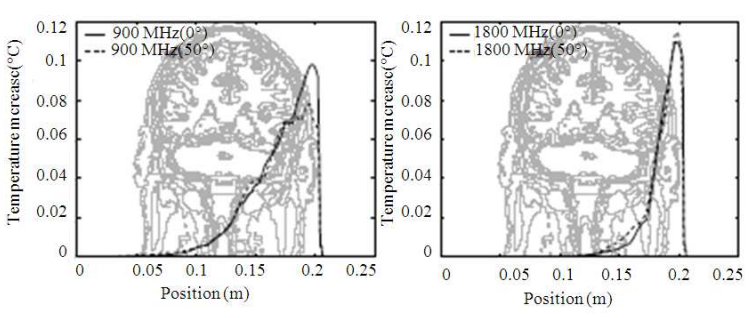

Fig. 18: Shows maximum temperature changes with different hand sets of orientations and frequency bands

$$
\begin{aligned}
& {[\nabla(\mathrm{k}(\mathrm{r}) \nabla \mathrm{T}(\mathrm{r}, \mathrm{t})+\rho(\mathrm{r}) \mathrm{SAR}(\mathrm{r})+\mathrm{Q}(\mathrm{r})-} \\
& \mathrm{B}(\mathrm{r})\left(\mathrm{T}(\mathrm{r}, \mathrm{t})-\mathrm{Tb}=\mathrm{C}(\mathrm{r}) \rho(\mathrm{r}) \frac{\mathrm{dT}(\mathrm{r}, \mathrm{t})}{\mathrm{dt}}\right]
\end{aligned}
$$

\section{CONCLUSION}

The important parameters affects the energy absorbed in the human head when exposed to radiation from mobile hand set is the distance between the antenna and head. Its observed that the power consumption at $1800 \mathrm{MHz}$ frequency is lower than that of $900 \mathrm{MHz}$ which is due to the maximum values of SAR are more at higher frequencies. The absorption is more in the skin, neck and outer layer of the head. Skull absorbs least energy due to poor conductivity. Also it is observed that SAR values are higher for the heterogeneous model of head when compared to homogeneous model. Future scope of our research is to implement entire model in $3 \mathrm{~d}$ and we are proposing metamaterial in the design of an antenna which is reducing SAR drastically.

\section{REFERENCES}

Choi, D.G., C.S. Shin, N. Kim and H.S. Shin, 2005. Design and SAR analysis of broadband PIFA with triple band. Proceedings of the Progress Electromagnetics Research Symposium, Aug. 226, Hangzhur, China, pp: 290-293. http://www.piers.org/piersonline/pdf/Vol1No3Pa ge290to293.pdf

Dey, S. and R. Mittra, 1997. A locally conformal finite-difference time-domain (FDTD) algorithm for modeling three-dimensional perfectly conducting objects. IEEE Microw. Guided Wave Lett, 7: 273-275. DOI: 10.1109/75.622536

Fayos-Fernandez, J., C. Arranz-Faz, A.M. MartinezGonzalez and D. Sanchez-Hernandez, 2006. Effect of pierced metallic objects on SAR distributions at $900 \mathrm{MHz}$. Bioelectromagnetics, 27: 337-353. DOI: 10.1002/BEM.20245 PMid:16724318

Fourie, A. and D. Nitch, 2000. SuperNEC: antenna and indoor-propagation simulation program. IEEE Antennas Propagat. Mag., 42 : 31-48. DOI: 10.1109/74.848946

Kawai, H. and K. Ito, 2004. Simple evaluation method of estimating local average SAR. IEEE Trans. Microwave Theory Techniques, 52: 20212029. DOI: 10.1109/TMTT.2004.832028

Liu, Y., Z. Liang and Z. Yang, 2008. Computation of electromagnetic dosimetry for human body using parallel fdtd algorithm combined with interpolation technique. Progress Electromagnetics Res., 82: 95-107. DOI: 10.2528/PIER08021603

Rhattoy, A., S. Bri and M. Audhuy-Peudecederf 2005. Coaxial antenna for microwave hyperthermia. J. Electromagnetic Waves Appli. 19: 1963-1971. DOI: 10.1163/156939305775570585

$\mathrm{Yu}$, W. and R. Mittra, 2004. Conformal FiniteDi.erence Time-Domain Maxwells Equations Solver: Software and Users Guide. $1^{\text {st }}$ Edn., Artech House, Boston, Mass, London, ISBN: 1580537316, pp: 330.

Zhu, Y., F. Gao, X. Yang, H. Shen and W. Liu et al., 2008. The effect of microwave emmision from mobile phones on neuron survival in rat central nervous system. Progress Electromagnetics Res., 82: 287-298. DOI: 10.2528/PIER08022813 